Radial and Nonradial Pulsations as Probes of Stellar Physics

ASP Conference Series, Vol. 259, 2002

C. Aerts, T.R. Bedding, $\mathcal{B}^{\prime} J$. Christensen-Dalsgaard, eds.

\title{
An Attempt at Mode Identification for V480 Tau
}

\author{
M. P. Di Mauro, J. Christensen-Dalsgaard, F. Pijpers \\ Theoretical Astrophysics Center, Aarhus, Denmark
}

J. Hao

Beijing Astronomical Observatory, Chin. Acad. of Sciences, P.R. China

\begin{abstract}
Spectroscopic observations of the $\delta$ Scuti star V480 Tau, obtained during the MUSICOS 1996 campaign (Hao et al., 2001), detected a large number of modes. In order to assess the information available from such data, we compare the observed spectrum of frequencies with the theoretical predictions obtained for models computed by including the effect of fast rotation on global oscillations.
\end{abstract}

\section{Observations}

The $\delta$ Scuti star V480 Tau, whose rotational velocity has been measured as $v \sin i=165 \mathrm{~km} / \mathrm{s}$ (Abt \& Morrell, 1995), was observed from 5 sites during a MUSICOS (Multi-Site COntinuous Spectroscopy) campaign in November December 1996 (Hao et al., 2001). The LSD (Least Square Deconvolution) method was employed to make good use of all the photospherical lines in the spectrum to produce only one set of time series with considerably higher $\mathrm{S} / \mathrm{N}$ ratio rather than several series of individual lines. Fourier analysis applied to the time series was able to identify a total of 16 modes, which range from 100 to $350 \mu \mathrm{Hz}$ in frequency and 5 to 23 in degree (Fig. 1).

\section{Oscillation Spectra Prediction}

To interpret oscillation observations of V480 Tau, we produced a grid of stellar models which include the spherically symmetric component of the effect of the centrifugal acceleration, by assuming uniform (solid-body) stellar rotation.

All the models have been calculated with the EFF equation of state (Eggleton et al., 1973) and OPAL opacities (Iglesias \& Rogers, 1996), Bahcall \& Pinsonneault (1992) nuclear cross sections and the mixing-length formalism (MLT) for convection. By the assumption of conservation of global angular momentum during the evolution, the equatorial rotational velocity decreases during the MSevolution, such as to become $\simeq 165 \mathrm{kms}^{-1}$ when it reaches the required values of $L$ and $T_{\text {eff }}$.

By assuming a value of luminosity $L / L_{\odot}=19.05 \pm 2.33$, a metallicity $Z=0.016 \pm 0.005$ and an effective temperature $T_{\text {eff }}=(7800 \pm 150) \mathrm{K}$ (Solano \& 

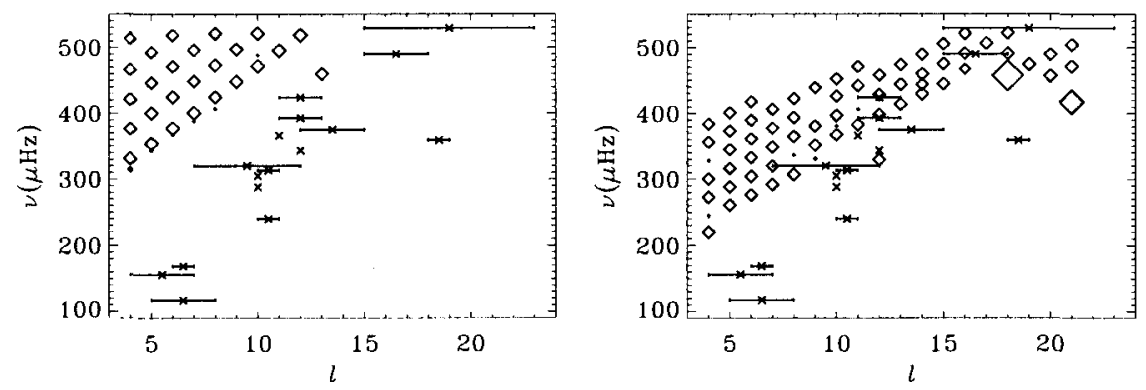

Figure 1. The observed frequencies (crosses) with the uncertainty in the determination of the harmonic degree $\ell$ are compared with the theoretical frequencies (diamonds) calculated, for prograde modes with $|m|=\ell$, for models with $M=1.9 M_{\odot}$ (left) and $M=2.3 M_{\odot}$ (right). The size of the open symbols indicates the relative surface amplitude of oscillation of the modes.

Fernley, 1997), this star can be identified in the HR diagram as being in the postmain-sequence phase of the evolution, with a mass in the range $M=1.9-2 M_{\odot}$.

The p-mode eigenfrequencies have been calculated by including the effects of the rotation on global oscillations by following a second-order perturbation approach as described by Christensen-Dalsgaard \& Thompson (1999). Amplitudes were estimated, relative to radial modes of the same frequency, such that the modes would have the same total energy (Christensen-Dalsgaard et al., 1995).

Theoretical oscillation spectra calculated for those models are not consistent with the observed frequencies, suggesting the necessity of a revision of the determination of the basic parameters of the star. A better match is obtained for a mass of $M=2.3 M_{\odot}$, such that the increases in luminosity and radius result in a decrease in the unperturbed frequencies, as well as in angular velocity and hence in the rotational splitting.

\section{References}

Abt, H. A., \& Morrell, N. I. 1995, ApJS, 99, 135

Bahcall, J. N., \& Pinsonneault, M. H. 1992, Rev. Mod. Phys., 64, 885

Christensen-Dalsgaard, J., Bedding, T. R., \& Kjeldsen, H. 1995, ApJ, 443, L29

Christensen-Dalsgaard, J., \& Thompson, M. J. 1999, A\&A, 350, 852

Eggleton, P. P., Faulkner, J., \& Flannery, B. P. 1973, A\&A, 23, 325

Hao, J., Kennelly, E. J, Catala, C., et al. 2001, to be submitted to A\&A

Iglesias, C. A., \& Rogers, F. J. 1996, ApJ, 464, 943

Solano, E., \& Fernley, J. 1997, A\&AS, 122, 131 